\title{
MINI-REVIEW
}

\section{Challenges in the Development, Deployment, and Regulation of Artificial Intelligence in Anatomic Pathology}

\author{
Jerome Y. Cheng, Jacob T. Abel, Ulysses G.J. Balis, David S. McClintock, and Liron Pantanowitz
}

From the Department of Pathology, University of Michigan, Ann Arbor, Michigan

\author{
Accepted for publication \\ October 23, 2020. \\ Address correspondence to \\ Jerome Y. Cheng, M.D., \\ Department of Pathology, Uni- \\ versity of Michigan, NCRC \\ Bldg. 35, Rm 30-1597, 2800 \\ Plymouth Rd., Ann Arbor, MI \\ 48109. E-mail: jeromech@ \\ med.umich.edu.
}

\begin{abstract}
Significant advances in artificial intelligence (AI), deep learning, and other machine-learning approaches have been made in recent years, with applications found in almost every industry, including health care. AI is capable of completing a spectrum of mundane to complex medically oriented tasks previously performed only by boarded physicians, most recently assisting with the detection of cancers difficult to find on histopathology slides. Although computers will likely not replace pathologists any time soon, properly designed AI-based tools hold great potential for increasing workflow efficiency and diagnostic accuracy in pathology. Recent trends, such as data augmentation, crowdsourcing for generating annotated data sets, and unsupervised learning with molecular and/or clinical outcomes versus human diagnoses as a source of ground truth, are eliminating the direct role of pathologists in algorithm development. Proper integration of AI-based systems into anatomic-pathology practice will necessarily require fully digital imaging platforms, an overhaul of legacy information-technology infrastructures, modification of laboratory/ pathologist workflows, appropriate reimbursement/cost-offsetting models, and ultimately, the active participation of pathologists to encourage buy-in and oversight. Regulations tailored to the nature and limitations of AI are currently in development and, when instituted, are expected to promote safe and effective use. This review addresses the challenges in AI development, deployment, and regulation to be overcome prior to its widespread adoption in anatomic pathology. (Am J Pathol 2021, 191: 1684-1692; https://doi.org/10.1016/j.ajpath.2020.10.018)
\end{abstract}

Recently, there has been much hype regarding the potential for machine learning (ML) and artificial intelligence (AI)based systems to replace or supplant physicians in many medical specialties, including anatomic pathology (AP). ${ }^{1,2}$ Consistent with this trend, several recent studies and reports in the general media have claimed that ML models are able to surpass human performance in various scenarios (Harvard Business Review, https://hbr.org/2019/10/aican-outperform-doctors-so-why-dont-patients-trust-it, last accessed October 26, 2020). ${ }^{1}$ Despite these stated capabilities for higher predictive accuracy, it is unlikely that AI-based tools will wholly replace physicians in the near future, given the frequent failure of AI-based solutions, which often exhibit unpredictable and/or incorrect results when confronted with data or patterns it has not encountered before. ${ }^{3}$ In such circumstances, ML algorithms operate in divergent or extrapolatory mode, in which spurious results are possible or even likely. ${ }^{3}$ Because of this intrinsic limitation, AI tools will see their greatest use and offer maximal benefit by providing supplemental assistance to providers in the immediate

\footnotetext{
Supported by institutional funding from the Department of Pathology, University of Michigan.

Disclosures: U.G.J.B. is a member of the advisory board of Inspirata; D.S.M. is a member of the scientific advisory board of Epredia; L.P. is a member of the medical advisory board of Ibex.

This article is part of a mini-review series on the applications of artificial intelligence and deep learning in advancing research and diagnosis in pathology.
} 
short-term. ${ }^{4}$ If medical specialties are willing to embrace this new approach, repetitive tasks currently performed by physicians and, specifically, pathologists may be suitable for supplemental AI-based assistance.

Indeed, amassed evidence to date demonstrates that a competent medical generalist, in cooperation with a properly developed AI tool, is able to surpass the performance of a medical specialist practicing without an AIbased solution. $^{5-7}$ This finding is congruent with those from a survey of 487 pathologists from 54 countries, of whom $71 \%$ indicated that AI tools could increase their diagnostic efficiency, although the majority of those queried thought that the diagnostic decision-making process should remain predominantly a human task. ${ }^{8}$ Caution is also necessary in the implementation of machine-based assistance in clinical settings, as pathologists' diagnostic decisions are prone to be influenced by AI, introducing novel sources of bias. ${ }^{6}$ A limited number of AI-based prediction tools have made their way into the market and have proved effective in practical clinical situations. ${ }^{1}$ However, while some studies highlight the capacity of these narrow AI algorithms to excel at a particular task, most neglect the broader complexity of conditions in clinical practice. In clinical practice, these tools would likely be exposed to a much wider array of heterogeneous cases and data patterns not seen before, leading such AI tools to either fail or exhibit lower accuracies than initially reported. A well-known example of this phenomenon is the subpar implementation of IBM Watson (IBM Watson Health, New York, NY).

Building a machine or deep-learning model entails a lengthy, weeks- or months-long process of development. It begins with the identification of a problem for which AI could be helpful, followed by data collection, data transformation, and ultimately, model training. ${ }^{10,11}$ If this process is successful, subsequent deployment of a reliably performing algorithm in a clinical setting (clinical laboratory or otherwise) undergoes rigorous validation studies (College of American Pathologists, https://www.cap.org/ member-resources/clinical-informatics-resources/how-tovalidate-ai-algorithms-in-anatomic-pathology, last accessed May 29, 2020). Approval for the marketing of commercial AI products also is obtained [eg, approval or clearance from the Conformité Européenne or the US Food and Drug Administration (FDA)] before they can be legally marketed for clinical use. ${ }^{12,13}$ The ultimate test is whether a product can be successfully integrated into pathologists' workflow. An early exemplar in this regard was the integration of FDA-cleared computerassisted automated Papanicolaou-smear screening in cytopathology. Unfortunately, there are still several hurdles to conquer before similar AI tools intended for use elsewhere in AP can be implemented. This review addresses challenges in AI development, deployment, and regulation that need to be overcome prior to widespread adoption in AP.

\section{Development Challenges}

\section{Problem Identification}

Pathologist buy-in is crucial for the successful adoption and integration of AI-based algorithms into practice. Such applications must have both clinical and practical utility in filling gaps or unmet needs without disrupting clinical workflow. Examples include rare-event identification, tumor-percentage calculation, mitosis detection, ${ }^{14}$ and other tasks found to be monotonous, tedious, or prone to higher interobserver variability. One notable example is Ki-67 index scoring in neoplasms, in which hundreds or thousands of tumor cells on tissue sections are counted in a repetitive task best suited for automated computation. The current manual process is unnecessarily time-consuming, compelling some pathologists to use an improvised workaround, at the expense of accuracy and reproducibility, for estimating the Ki-67 index via light microscopy through a process referred to as eyeballing. ${ }^{15}$ This example clearly shows a situation in which AI tools can be developed, with pathologists' input, to improve both diagnostic quality and pathologists' efficiency. However, if AI tools are developed merely for novelty or intellectual appeal, they have a low likelihood of being utilized in routine clinical practice. Thus, AI startups need to be cautious of the shiny-object syndrome and to avoid the trap of searching for the elusive killer application. Instead, these companies should focus on tools integral to pathologists' work, with the recognition that some of the low-hanging fruit in this regard may be relatively mundane, but nonetheless important, tasks.

\section{Data Set Curation and Annotation}

Determining a data set for use in AI-algorithm generation does not follow a one-size-fits-all approach and by itself can be a complicated task. For example, convolutional neural networks typically require large-scale training sets, from hundreds or thousands of slides, in order to achieve high model accuracy, significant performance gains, and increased generalizability. ${ }^{16}$ Conversely, in cases involving transfer learning (Model Development and Training), smallscale data sets consisting of $<100$ digital slides may suffice. ${ }^{17}$ For rare diseases, only an extremely limited number of slides may be available, leading some to simulate largerscale data sets through data-augmentation techniques. Therefore, the actual number of slides needed for a given AI task varies depending on the problem at hand.

Adding more categories to an AI-classification task will also necessarily increase the number of slides/images necessary for algorithm training (eg, classifying two types of cancer requires fewer training samples than does building a model for classifying five types of cancer). Publicly available data sets can be helpful for supplementing locally curated image repositories; however, partially due to 
confidentiality, copyright, and budgetary issues, few such data sets are available in pathology. ${ }^{18}$ The Cancer Genome Atlas is one publicly available data set that offers a substantial number of cases with digital slides and molecular metadata. ${ }^{19,20}$ Unfortunately, The Cancer Genome Atlas has limited cases from many diagnostic subsets, with numbers often insufficient for training clinical-grade histopathology AI models. Another helpful, albeit limited, source of data sets is in the form of public challenges offered for developing deep-learning algorithms. ${ }^{21}$

After the data-collection step, the next task in deep-learning algorithm development is labeling the data (eg, malignant versus benign, necrosis versus fibrosis). Annotation types vary based on deep-learning algorithm type, as follows:

i) Classification problems: images and image labeling

ii) Image segmentation: image and pixel-level labeling (ground truth map)

iii) Object detection and classification: bounding box coordinates and class of image within the bounding box

iv) Weakly supervised methods: slide-level classifications (versus labeling each image tile)

In one published study involving thousands of digital slides and multiple-instance learning in which all tiles that belonged to the same slide were associated with the same slide-level classification, it was still possible to attain veryhigh-accuracy scores. $^{16}$ The expert-driven annotation process is time-consuming and laborious, especially considering the large number of images and significant person-hours required for review and annotation. Furthermore, the accuracy of trained models may be affected by variability in annotation performance, particularly if a task is difficult (eg, programmed cell death ligand 1 staining of tumor cells versus macrophages). This expert-driven annotation bottleneck has proved to be expensive for manufacturers; however, AI-assisted annotation techniques are emerging as a potential solution for reducing the manual effort needed for labeling image data sets (Nvidia, https:// developer.nvidia.com/blog/annotation-transfer-learning-claratrain, last accessed July 4, 2020).

Crowdsourcing is another proved and viable method of labeling sizable data sets (eg, through quantity-based payments, gamification, and marketing elements). ${ }^{22}$ ImageNet, arguably the most popular publicly available image data set, leveraged crowdsourcing through Amazon's Mechanical Turk to label over 14 million images from $21,000+$ classes. ${ }^{23}$ However, crowdsourcing should be used with caution, given the inherent noise introduced by such diverse annotation methods and variable expertise of those involved. For those who prefer to annotate images themselves, many standalone tools (eg, QuPath, ${ }^{24}$ designed for pathologists) and web-based tools (eg, Lionbridge AI, https://lionbridge.ai/articles/image-annotation-tools-for-com puter-vision, last accessed October 26, 2020) are available, although they are not without potential difficulties, as some are known to have user-interface design issues.

\section{Model Development and Training}

A significant amount of trial and error is needed for developing models with superior performance metrics. The model design of a deep-learning system can have a major bearing on bias and thereby the success of the predictions of an algorithm. ${ }^{25}$ The following points highlight the most common steps involved in model development and training:

i) Splitting the data into training, testing, and holdout data sets $^{26}$

ii) Selecting an ML framework (eg, Pytorch, Tensorflow)

iii) Choosing the best ML technique (eg, convolutional neural networks for images) or building your own model

iv) Selecting the learning method (eg, transfer learning)

v) Evaluating the model through performance metrics (eg, area under the curve, f1 score, accuracy)

vi) Fine-tuning hyperparameters

A data set that is sufficiently large and comprises digital slides of the diagnoses of interest may not always be available for training. In this case, one can use transfer learning, a machine-learning technique that leverages knowledge gained from one model and applies it to a different model, in many cases still maintaining high accuracy. Models pretrained on public data sets, such as the ImageNet data set consisting of everyday objects (eg, trees, pens, animals), are popular options for transfer learning and can be effective in making predictions about new subject matter. ${ }^{23,27}$ Within pathology, one can surmise that transfer learning will become more effective after models trained on large-scale histopathologic data sets become publicly available. In this case, the availability of large-scale data sets that cover all of the variances found in diagnoses of interest would be expected to lead to model outcomes with higher accuracy versus transfer-learning algorithms trained on small-scale data sets. However, subsequent clinical validation still requires data sets independent from the one utilized for analytical validation of the algorithm. Currently, deep-learning-based algorithms are more convenient for development in nonmedical fields, given that pretrained models are readily available and can be used immediately and with little modification.

\section{Hardware and Cost}

AI training is a time-consuming process when large-scale data sets and complex models are involved. ${ }^{16}$ While the use of advanced hardware with multiple graphical processing units can speed up training, it can become prohibitively expensive. When building a high-end deep-learning workstation, it is beneficial to choose faster central processing units, increased system and graphics processing unit random-access memory, and readily accessible and adequate storage space. For those who plan to train simpler AI models using smaller-scale data sets, sub- $\$ 1000$ 
deep-learning workstations can be used. Additionally, lowcost graphics processing units can be added to most personal computers for computationally less-demanding projects, lessening the barriers initially encountered when exploring AI tools.

\section{Deployment Challenges}

Multiple deployment challenges must be overcome prior to the implementation of a pathology AI system into a clinical work environment. To start, fundamental requirements such as defined business-use cases and pathologists' assurances for using the AI system must be met before significant time, effort, and money are spent on AI-associated software, hardware, and implementation efforts. The transition from glass to digital workflows in AP must also be considered, as departments will require new digital pathology equipment, image-management systems, increased data-management and storage capabilities, and additional employees to accommodate for the changes in workflow. Systems will need to work with locally generated pathology-laboratory data hosted on established institutional informationtechnology (IT) infrastructures, through either on-premises or cloud-based computing solutions.

Finally, one must acknowledge pathologists' relative inexperience with $\mathrm{AI}$ and understand the range of questions that every department will face prior to the adoption of AI. One example of the failed adoption of a promising health care AI technology that did not take these factors into account involved an academic medical center spending approximately $\$ 62$ million on IBM Watson to assist in clinical decision making. Unfortunately, since IBM Watson was not previously used by the medical center for a single clinical decision on actual patients, the implementation was afflicted by unexpected costs and learning problems from unstructured data. ${ }^{28}$

\section{Pathologist Buy-In and Transitioning to a Digital Workflow}

First, one must gain pathologist buy-in for major changes to what is admittedly a century-old workflow. Given that change does not happen overnight, change-management issues for pathologist end-users should be anticipated separate from the technical hurdles. To begin with, institutional leadership must commit to the adoption of both digital pathology and AI within AP, demonstrating to pathologists how the long-term benefits of digital workflows outweigh their risks. Historically, resistance from pathologists to adopt digital pathology and AI may be attributed to a lack of trust, technophobia, uncertain liability, and concerns that AI may one day replace physicians. ${ }^{2}$ The fact that many AI tools are a black box to the user further compounds clinicians' reluctance to adopt AI. Instead, explainable AI solutions now exist, offering a promising alternative where ML-based methods are generated in order to be more easily understood by humans, thus providing pathologists with clear explanations as to how predictions are generated. ${ }^{29}$

Fundamentally, the transition to digital pathology must occur before AI deployment is possible in AP. Implementing digital pathology has a number of proved use cases, including primary and secondary clinical diagnosis, telepathology, convenient slide sharing, robust research data set creation, and pathology education/teaching. ${ }^{30}$ Digital images can be acquired in multiple ways, from simple cameras on microscopes to complex whole-slide imaging systems, and most recently, augmented-reality microscopes, in which digital images are overlaid on glass-slide fields of view. ${ }^{31}$ With recently developed high-throughput whole-slide imaging systems, faster and less expensive bulk data storage, increased network bandwidth, and improved informationsystems interoperability, digital pathology workflows are now a feasible option for pathology departments. However, even with these advances, only a handful of pathology practices currently sign out most of their cases digitally. ${ }^{32}$ Overall, there has been much resistance globally to go fully digital, ${ }^{33}$ drawing parallels to clinicians resisting the change from paper charts to electronic health records or radiologists from film to digital scans. ${ }^{34}$ Fortunately, the current hype and ensuing desire to leverage AI within medicine have bolstered the business case for implementing digital pathology into many clinical laboratories and pathology practices.

\section{IT Infrastructure: Cloud Computing versus on-Premises Solutions}

The decision of whether to use a cloud-based or on-premise AI solution depends on several factors, namely one's preferred workflow, how often the tools will get used, software and hardware costs, and the inclination of one's IT cybersecurity risk group to allow the use of cloud-based solutions. When considering a cloud-based AI solution, cost

Table 1 Example of an AI Validation Study Parameters for Clinical Practice

\begin{tabular}{llcc}
\hline Phase & Calibration & Analytical validation & Clinical validation \\
\hline Rationale & $\begin{array}{c}\text { Check that AI works as } \\
\text { specified }\end{array}$ & $\begin{array}{c}\text { Check that AI } \\
\text { consistently } \\
\text { performs as expected }\end{array}$ & $\begin{array}{c}\text { Confirm that AI works } \\
\text { safely with routine } \\
\text { workflow } \\
\text { Number WSIs }\end{array}$ \\
\hline
\end{tabular}

WSIs, whole-slide images. 


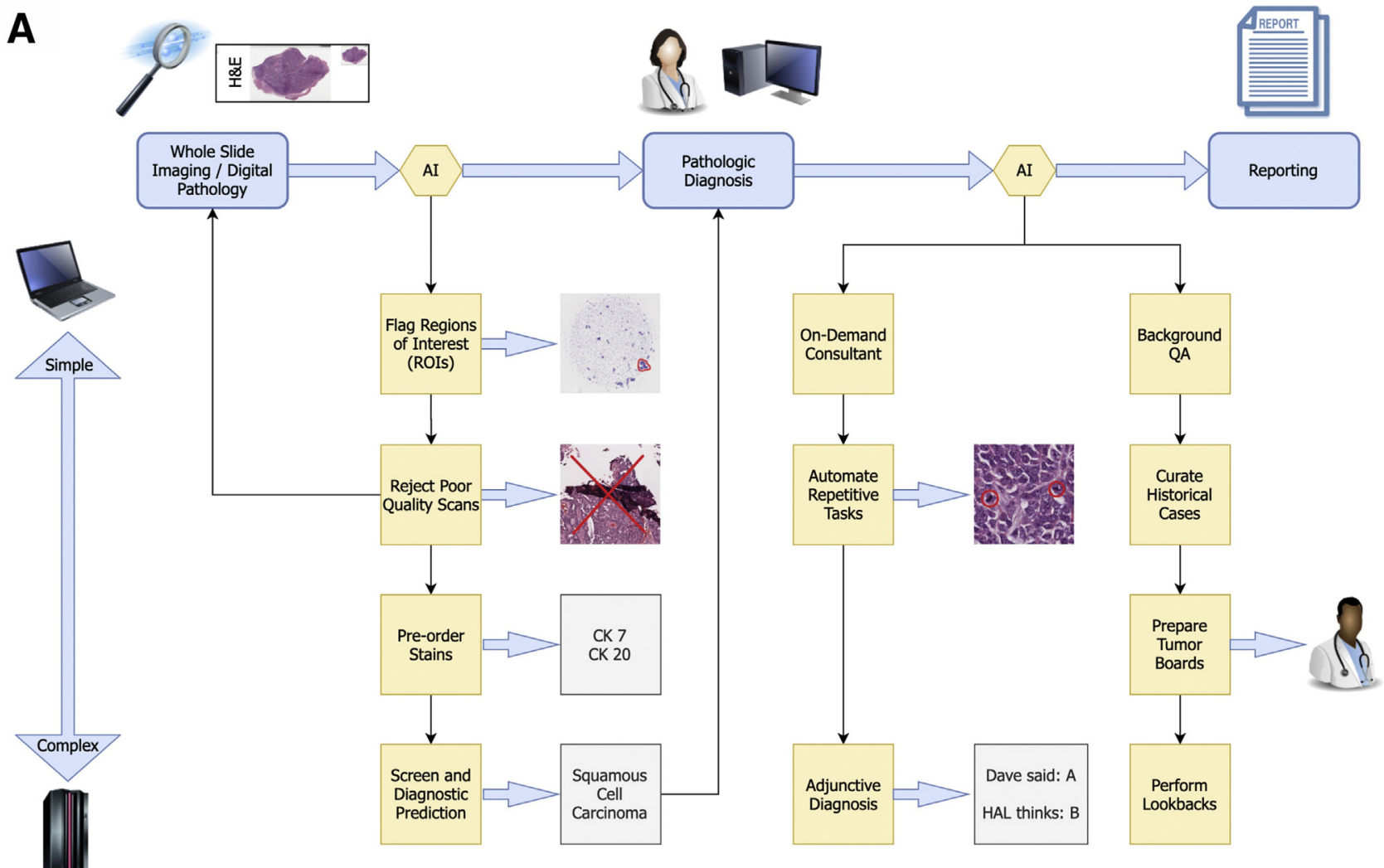

B

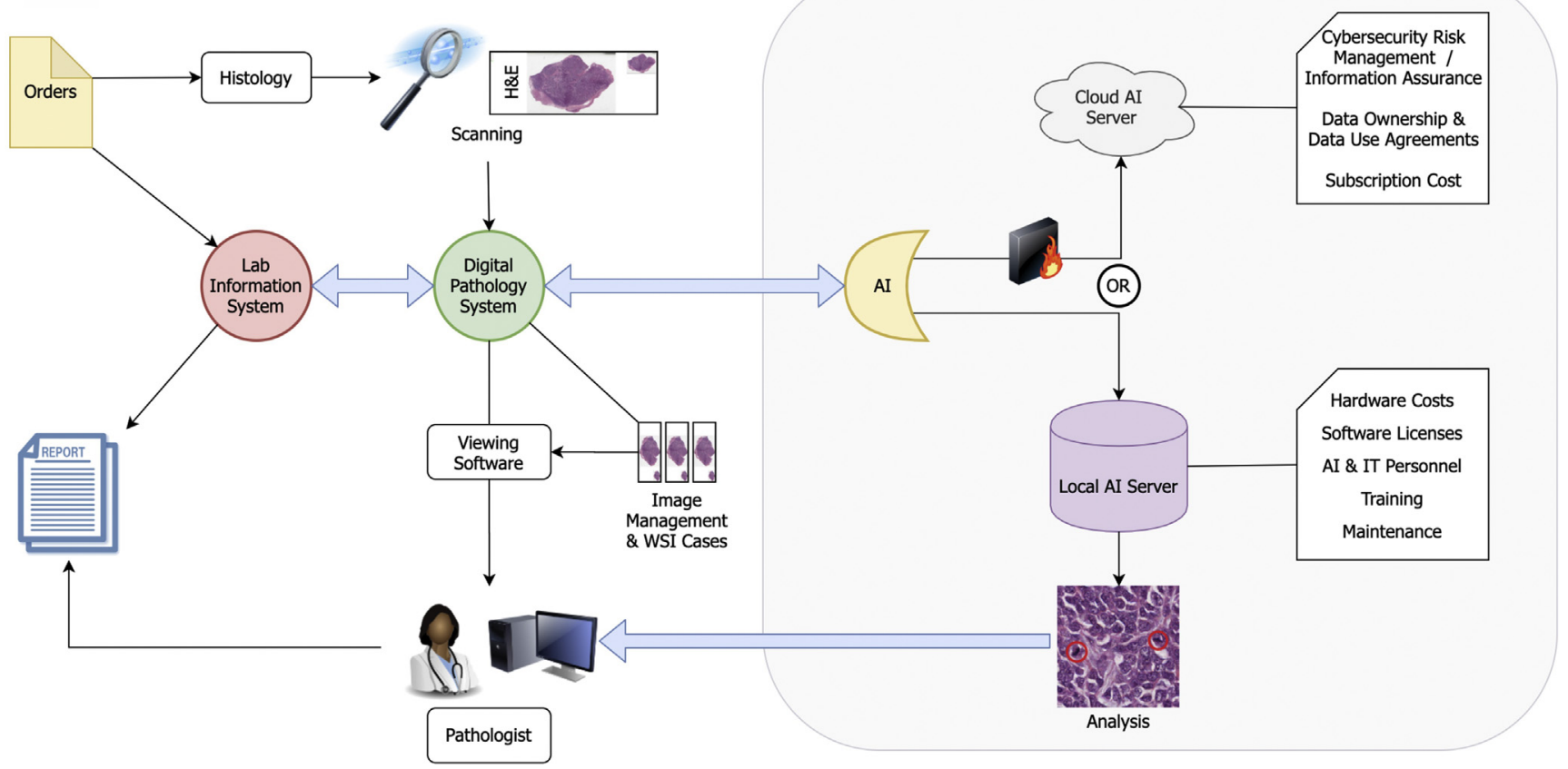

Figure 1 A: Embedding AI into anatomic pathology clinical workflows. The top horizontal axis represents AI algorithm execution before, during, or after the pathologist diagnostic process. These algorithms can run automated in the background or be manually activated by the pathologist when needed. The left vertical axis represents how AI algorithms differ in relative complexity, ranging from simple mitotic counts to complex rendering of adjunctive diagnoses as an "independent" observer. B: Proposed integration of AI into the AP laboratory information-technology (IT) architecture. In this scheme, the digital pathology system is connected to a separate server running AI analysis pipelines, which can either be local/on-premises or interfaced remotely as a cloud-based solution. Whole-slide imaging (WSI) data are submitted to this server, running the algorithms and returning results to the digital pathology system and to the pathologist. AI algorithm findings are interpreted by the pathologist and subsequently incorporated into the pathology report. CK, cytokeratin; H\&E, hematoxylin and eosin; QA, quality assurance. 
is one of the primary issues, with vendors often charging for graphics processing unit computational use on a usage basis ${ }^{35}$ in addition to any storage costs required. Furthermore, the potential for a data breach and/or inappropriate use of patient data increases with each external handoff of the data - a solid business associate's agreement, full endto-end encryption, and clear data-use agreements are essential for cloud-based systems. However, even with these potential barriers, the ability to outsource the AI algorithm development and expertise, in addition to the external management of hardware and software maintenance, may make these solutions the preferred option.

Conversely, purchasing and implementing an onpremises solution may be optimal if a laboratory has access to a robust IT infrastructure and plans to perform frequent and computationally intensive AI calculations. Moreover, some AI algorithms can function adequately on low-cost hardware, allowing for a smaller initial deployment with incremental adoption as AI needs increase. The abilities to fully control the data pipeline and custom-configure an AI solution to one's clinical workflow are also added advantages, especially considering that one size rarely fits all. From a cybersecurity perspective, there is fundamentally decreased risk with on-premises solutions because these, by definition, restrict data from leaving the institution. Overall, the decision to implement a cloud-based or on-premises solution comes down to cost, security, and departmental preferences. ${ }^{36}$

\section{Pathologists' Lack of Experience with AI}

Most pathology departments have never used AI for surgical pathology in their typical case-signout routine,${ }^{20}$ leading to a number of concerns when one begins implementing ML-based software for this purpose. The following issues are commonly encountered:

\section{What Is the Right Evidence Standard for AI To Be Embedded in Practice?}

There is no clear evidence standard for using AI in pathology at this time. Whether AI use in routine clinical practice will be based on algorithm performance, regulatory approval, validation with large-scale clinical trials, or published evidence in peer-reviewed scientific literature remains to be determined.

\section{What Is Required for Clinical Validation Prior to Using AI for diagnostic purposes?}

Guidelines on how best to validate whole-slide imaging and quantitative image analysis for clinical use are available. However, no recommendations on AI clinical validation are currently available. Table 1 provides proposed criteria for AI validation in pathology laboratories.
What Is the Ideal Workflow when Implementing AI in Clinical Practice?

Depending on the need, AI tools can be embedded within a pathology laboratory workflow before or after pathologist case signout (Figure 1A). Prior to pathologists' receiving cases for review, an AI tool can be used for triaging cases (eg, AI-detected cancer cases receive higher priority) or for assisting with rare-event screening (eg, microorganism identification). Pathologists can also trigger AI tools to perform specific tasks after case review (eg, for counting mitotic figures for tumor grading). Finally, AI software can also run in the background, continually performing quality assurance and other tasks (eg, cytologic-histologic correlation). The generation of a successful AI workflow for meeting one's needs ultimately depends on seamless interoperability between AI, digital pathology, and laboratory information systems (Figure 1B).

\section{What Model Do Pathology Laboratories Use for Paying AI Vendors?}

Unfortunately, given that few pathology laboratories use AI in routine practice, few data exist regarding a best payment model for AI services. Current prevailing options include subscription, akin to cloud-based software as a service, or pay-per-click models.

\section{What Is the Business-Use Case for Deploying AI?}

Whether improvements in efficiency and accuracy for pathologists will generate sufficient return on investment to drive the widespread adoption of AI tools is currently unknown. Ultimately, more data from clinical practice are needed for defining the added value of AI tools in pathology practices before this question can be fully answered.

\section{In Academic Medical Centers, Should Residents or Fellows Be Allowed To Use AI, or Is This Cheating?}

Trainee de-skilling, in which excessive reliance on AI assistance may provide fewer opportunities for trainees to develop their ability to discern different types of lesions on histopathology, is certainly a potential future concern with AI use. However, when used as an adjunctive tool (eg, as an extra set of eyes) and not as a replacement for pathologists, AI has the ability to increase the efficiency and accuracy of clinical diagnoses. Therefore, pathologists, starting early in residency, should be trained on the proper use, limitations, and pitfalls of AI tools similar to how they learn to wisely use special and immunohistochemistry analysis stains, fluorescence in situ hybridization, molecular assays, and other ancillary studies. 


\section{Regulatory Challenges}

AI-based algorithms may seem much more capable than they really are, with some of these tools perceived as black boxes due to human difficulty in comprehending exactly how predictions are made. Even with methods such as gradient saliency maps ${ }^{37}$ and filter visualization techniques that may help pathologists to understand AI-based predictions, humans cannot fully understand how millions of parameters contribute to a decision, leading to potential biases, misuse, and misdiagnoses. Therefore, a regulatory pathway for AI is required for promoting safe, proper, and effective use of these algorithms while concurrently taking into account their intricacies and limitations. The issue of how to properly regulate AI will become increasingly important as more algorithms are used in clinical practice. Regulatory approval should aim to not only mitigate potential harm, but also strike a proper balance between defining risk and benefits, developing effective validation standards, and promoting innovation. ${ }^{38}$ The current landscape for AI-device regulation is evolving, with regulatory bodies such as the FDA, the European Union Conformité Européenne, and the Centers for Medicare and Medicaid Services not fully prepared for the influx of AI into medicine. Consequently, devices enabled with ML capabilities are being regulated using prior, and arguably outdated, standards for tests and medical devices. ${ }^{39}$

\section{FDA}

Getting FDA market approval or clearance is a long, expensive, and stringent process. The difficulty of submissions depends on the type of application into which a device falls-premarket approval, 501(k) clearance, or de novo granted. While the FDA has approved several AI algorithms for medical use, ${ }^{1}$ it recognizes that current regulations were not designed for ML and adaptive technologies, most of which would end up necessitating frequent, if not continuous, premarket review for algorithm modifications under the current guidelines (FDA, https://www.fda.gov/ medical-devices/software-medical-device-samd/artificial-intelli gence-and-machine-learning-software-medical-device, last accessed Oct 26, 2020). With this in mind, the FDA is currently drafting a set of new regulations to better address the safety and efficacy of AI-enabled devices in the market (FDA, https://www.fda.gov/media/122535/ download, last accessed May 29, 2020).

\section{European Union Conformité Européenne}

With a focus on health and safety (European Commission, https://ec.europa.eu/growth/sectors/medical-devices_en, last accessed May 29, 2020), Conformité Européenne marking for medical devices can be acquired for AI-capable devices through the in vitro diagnostic medical device directive and medical device directive. If obtained, Conformité Européenne mark approval means that an AI-based system can be legally marketed within the European Union and European Free Trade Association member states. The latest in vitro diagnostic medical device directive that went into effect on May 25, 2017, requires that scientific validity, analytical performance, and clinical performance be demonstrated prior to approval. ${ }^{12}$ Of note, medical device directive will be replaced by medical device regulation in May 2021, and in vitro diagnostic medical device directive will be replaced by the in-vitro diagnostic regulation in May 2022 (European Commission, https:/lec.europa.eu/growth/ sectors/medical-devices_en, last accessed May 29, 2020). In order to address the growing impact of AI, the European Commission recently published a white paper on AI that included broad proposals concerning AI regulation (European Commission, https://ec.europa.eu/ info/sites/info/files/commission-white-paper-artificial-intell igence-feb2020_en.pdf, last accessed October 26, 2020).

\section{Centers for Medicare and Medicaid Services/CLIA}

In the United States, the Centers for Medicare and Medicaid Services regulate laboratory testing through the Clinical Laboratory Improvement Amendments (CLIA). CLIA stipulates that all tests performed on human tissue must undergo proper validation by the performing laboratory prior to their introduction for clinical use, regardless of the FDA status of the test (National Archives: Definitions. Code of Federal Regulations, Title 42, Chapter IV, Subchapter G, Subpart A, Section 493.2, https://www.ecfr.gov/cgi-bin/text$i d x ?$ node $=$ se42.5.493_12\&rgn $=$ div8, $\quad$ last accessed August 6, 2020). Currently, there are no specific CLIA regulations for validating $\mathrm{AI}$ algorithms, nor do the Centers for Medicare and Medicaid Services/CLIA have a published opinion on whether AI algorithms fall into the category of laboratory-developed tests. For AI-algorithm validation, most pathologists would naturally look to typical laboratory-validation methodologies, as defined by CLIA, Centers for Medicare and Medicaid Services, and its deemed agencies (eg, the College of American Pathologists). However, this is not the case for most (nonpathologist) clinicians and researchers, given that, from the authors' experiences, most nonlaboratorians are not familiar with CLIA and thus would not seek laboratory regulations for guidance. Furthermore, given that AI is performed on data and not on human-derived tissue, one can argue that AI algorithms do not apply as laboratory tests. Therefore, given these ambiguities as to how to classify AI tools, in addition to a lack of clear guidelines surrounding their regulation, no firm recommendations can be made regarding how AI algorithms should be definitively validated at this time.

\section{Conclusion}

Currently, AI has tremendous potential to improve the practice of pathology through streamlining workflow (eg, 
weeding out negative cases), reducing errors (eg, screening for hard-to-find lesions, resolving atypical diagnoses), improving reproducibility (eg, standardizing diagnostic decision making), and conveying predictions not possible with a microscope alone. However, AI use for clinical work should be affordable, practical, interoperable, explainable, generalizable, manageable, and reimbursable. Additionally, the domain expertise of pathologists is crucial for optimal expert system design and development. ${ }^{40}$ With the current limited clinical experience and uncertainty on how to embed AI technology into clinical practice, caution must be exercised to avoid unwanted consequences when deploying AI. Finally, only with pathologist buy-in, standardized recommendations and guidelines, and seamless interoperability of AI tools with current information systems will AI be adopted and used safely in AP laboratories.

\section{References}

1. Topol EJ: High-performance medicine: the convergence of human and artificial intelligence. Nat Med 2019, 25:44-56

2. Ahuja AS: The impact of artificial intelligence in medicine on the future role of the physician. PeerJ 2019, 7:e7702

3. Oh S, Kim JH, Choi S-W, Lee HJ, Hong J, Kwon SH: Physician confidence in artificial intelligence: an online mobile survey. J Med Internet Res 2019, 21:e12422

4. He J, Baxter SL, Xu J, Xu J, Zhou X, Zhang K: The practical implementation of artificial intelligence technologies in medicine. Nat Med 2019, 25:30-36

5. Pesapane F, Tantrige P, Patella F, Biondetti P, Nicosia L, Ianniello A, Rossi UG, Carrafiello G, Ierardi AM: Myths and facts about artificial intelligence: why machine- and deep-learning will not replace interventional radiologists. Med Oncol Northwood Lond Eng1 2020, 37:40

6. Kiani A, Uyumazturk B, Rajpurkar P, Wang A, Gao R, Jones E, Yu Y, Langlotz CP, Ball RL, Montine TJ, Martin BA, Berry GJ, Ozawa MG, Hazard FK, Brown RA, Chen SB, Wood M, Allard LS, Ylagan L, $\mathrm{Ng}$ AY, Shen J: Impact of a deep learning assistant on the histopathologic classification of liver cancer. NPJ Digit Med 2020, 3:23

7. Niazi MKK, Parwani AV, Gurcan MN: Digital pathology and artificial intelligence. Lancet Oncol 2019, 20:e253-e261

8. Sarwar S, Dent A, Faust K, Richer M, Djuric U, Van Ommeren R, Diamandis P: Physician perspectives on integration of artificial intelligence into diagnostic pathology. Npj Digit Med 2019, 2:28

9. Lee W-S, Ahn SM, Chung J-W, Kim KO, Kwon KA, Kim Y, Sym S, Shin D, Park I, Lee U, Baek J-H: Assessing concordance with watson for oncology, a cognitive computing decision support system for colon cancer treatment in Korea. JCO Clin Cancer Inform 2018, 2:1-8

10. LeCun Y, Bengio Y, Hinton G: Deep learning. Nature 2015, 521: 436-444

11. Fu W, Menzies T: Easy over hard: a case study on deep learning. Proceedings of the 2017 11th Joint Meeting on Foundations of Software Engineering (ESEC/FSE 2017); 2017. pp. 49-60

12. García-Rojo M, De Mena D, Muriel-Cueto P, Atienza-Cuevas L, DomínguezGómez M, Bueno G: New European Union regulations related to whole slide image scanners and image analysis software. J Pathol Inform 2019, 10:2

13. Harrington SG, Johnson MK: The FDA and artificial intelligence in radiology: defining new boundaries. J Am Coll Radiol JACR 2019, 16:743-744

14. Saha M, Chakraborty C, Racoceanu D: Efficient deep learning model for mitosis detection using breast histopathology images. Comput Med Imaging Graph 2018, 64:29-40
15. Niazi MKK, Senaras C, Pennell M, Arole V, Tozbikian G, Gurcan MN: Relationship between the Ki67 index and its area based approximation in breast cancer. BMC Cancer 2018, 18:867

16. Campanella G, Hanna MG, Geneslaw L, Miraflor A, Werneck Krauss Silva V, Busam KJ, Brogi E, Reuter VE, Klimstra DS, Fuchs TJ: Clinical-grade computational pathology using weakly supervised deep learning on whole slide images. Nat Med 2019, 25:1301-1309

17. Jones AD, Graff JP, Darrow M, Borowsky A, Olson KA, GandourEdwards R, Datta Mitra A, Wei D, Gao G, Durbin-Johnson B, Rashidi HH: Impact of pre-analytical variables on deep learning accuracy in histopathology. Histopathology 2019, 75:39-53

18. Hipp JD, Sica J, McKenna B, Monaco J, Madabhushi A, Cheng J, Balis UJ: The need for the pathology community to sponsor a whole slide imaging repository with technical guidance from the pathology informatics community. J Pathol Inform 2011, 2:31

19. Tomczak K, Czerwińska P, Wiznerowicz M: The Cancer Genome Atlas (TCGA): an immeasurable source of knowledge. Contemp Oncol Poznan Pol 2015, 19:A68-A77

20. Bera K, Schalper KA, Rimm DL, Velcheti V, Madabhushi A: Artificial intelligence in digital pathology - new tools for diagnosis and precision oncology. Nat Rev Clin Oncol 2019, 16:703-715

21. Hartman D, Van der Laak JAWM, Gurcan M, Pantanowitz L: Value of public challenges for the development of pathology deep learning algorithms. J Pathol Inform 2020, 11:7

22. Hughes AJ, Mornin JD, Biswas SK, Beck LE, Bauer DP, Raj A, Bianco S, Gartner ZJ: Quanti.us: a tool for rapid, flexible, crowdbased annotation of images. Nat Methods 2018, 15:587-590

23. Deng J, Dong W, Socher R, Li L-J, Li K, Li F-F: ImageNet: a largescale hierarchical image database. 2009 IEEE Conference on Computer Visual Pattern Recognition (CVPR 2009), 2009 June 22-24. Miami, FL, IEEE Computer Society, 2009. pp. 248-255

24. Bankhead P, Loughrey MB, Fernández JA, Dombrowski Y, McArt DG, Dunne PD, McQuaid S, Gray RT, Murray LJ, Coleman HG, James JA, Salto-Tellez M, Hamilton PW: QuPath: open source software for digital pathology image analysis. Sci Rep 2017, 7:16878

25. Eklund M, Kartasalo K, Olsson H, Ström P: The importance of study design in the application of artificial intelligence methods in medicine. NPJ Digit Med 2019, 2:101

26. Rashidi HH, Tran NK, Betts EV, Howell LP, Green R: Artificial intelligence and machine learning in pathology: the present landscape of supervised methods. Acad Pathol 2019, 6. 237428951987308

27. Komura D, Ishikawa S: Machine learning approaches for pathologic diagnosis. Virchows Arch Int J Pathol 2019, 475:131-138

28. Schmidt C: MD Anderson breaks with IBM Watson, raising questions about artificial intelligence in oncology. J Natl Cancer Inst 2017, 109

29. Tosun AB, Pullara F, Becich MJ, Taylor DL, Fine JL, Chennubhotla SC: Explainable AI (xAI) for anatomic pathology. Adv Anat Pathol 2020, 27:241-250

30. Volynskaya Z, Evans AJ, Asa SL: Clinical applications of wholeslide imaging in anatomic pathology. Adv Anat Pathol 2017, 24: 215-221

31. Chen P-HC, Gadepalli K, MacDonald R, Liu Y, Kadowaki S, Nagpal K, Kohlberger T, Dean J, Corrado GS, Hipp JD, Mermel CH, Stumpe MC: An augmented reality microscope with real-time artificial intelligence integration for cancer diagnosis. Nat Med 2019, 25: $1453-1457$

32. Retamero JA, Aneiros-Fernandez J, del Moral RG: Complete digital pathology for routine histopathology diagnosis in a multicenter hospital network. Arch Pathol Lab Med 2020, 144:221-228

33. Evans AJ, Salama ME, Henricks WH, Pantanowitz L: Implementation of whole slide imaging for clinical purposes: issues to consider from the perspective of early adopters. Arch Pathol Lab Med 2017, 141:944-959

34. Ajami S, Bagheri-Tadi T: Barriers for adopting electronic health records (EHRs) by physicians. Acta Inform Med 2013, 21:129-134 
35. Langmead B, Nellore A: Cloud computing for genomic data analysis and collaboration. Nat Rev Genet 2018, 19:208-219

36. Matthews D: Supercharge your data wrangling with a graphics card. Nature 2018, 562:151-152

37. Pasa F, Golkov V, Pfeiffer F, Cremers D, Pfeiffer D: Efficient deep network architectures for fast chest X-ray tuberculosis screening and visualization. Sci Rep 2019, 9:6268
38. Allen TC: Regulating artificial intelligence for a successful pathology future. Arch Pathol Lab Med 2019, 143:1175-1179

39. Minssen T, Gerke S, Aboy M, Price N, Cohen G: Regulatory responses to medical machine learning. J Law Biosci 2020, 7: 1 saa002

40. Huss R, Coupland SE: Software-assisted decision support in digital histopathology. J Pathol 2020, 250:685-692 